\title{
Avaliação do comportamento motor de crianças entre zero a 12 meses incompletos em região periférica na cidade de Santos
}

\section{Evaluation of motor behavior in children between zero and under 12 months in a peripheral region of Santos}

\author{
Andréa Felner Navajas ${ }^{1}$, Silvana Maria Blascovi-Assis ${ }^{2}$ \\ http://dx.doi.org/10.11606/issn.2238-6149.v27i3p246-253
}

\begin{abstract}
Navajas AF, Blascovi-Assis SM. Avaliação do comportamento motor de crianças entre zero a 12 meses incompletos em região periférica na cidade de Santos. Rev Ter Ocup Univ São Paulo. 2016 set.-dez.;27(3):246-53.

RESUMO: O desenvolvimento infantil tem sido objeto de estudo frequente considerando os diferentes fatores de risco apontados na literatura. Este estudo teve como objetivo avaliar o comportamento motor de crianças nascidas a termo na Zona Noroeste de Santos. Participaram 50 crianças, na faixa etária entre zero e 12 meses incompletos, de ambos os sexos, que foram avaliadas pela Escala de Desenvolvimento do comportamento da criança no primeiro ano de vida. Os participantes foram divididos em três grupos: grupo de desenvolvimento normal (GDN), grupo de desenvolvimento regular (GDR), grupo risco ou atraso (GRA). Os resultados mostraram que $48 \%$ das crianças avaliadas foram classificadas com risco ou atraso para o desenvolvimento. Pode-se concluir que crianças que vivem em comunidades nas regiões periféricas devem participar de programas de triagem e intervenção para que possam ter minimizadas as chances de apresentarem riscos ao desenvolvimento.
\end{abstract}

DESCRITORES: Desenvolvimento infantil; Avaliação; Prevenção primária; Criança.
Navajas AF, Blascovi-Assis SM. Evaluation of Motor Behavior in children between zero and under 12 months in a peripheral region of Santos. Rev Ter Ocup Univ São Paulo. 2016 Sept.Dec.;27(3):246-53.

ABSTRACT: Child development has been a subject of frequent study considering the various risk factors cited in the literature. The aim of this study was to evaluate the motor behavior of children born at term in the Northwest region of Santos. Fifty children, of both sexes, aged between zero and under 12 months participated in the study and were evaluated by the Child Behavior Development Scale in the first year of life. The participants were divided into three groups: normal development (ND), regular development (RD), risk or delay group (RDG). The results showed that $48 \%$ of the children evaluated were classified with risk or delayed development. We concluded that children who live in peripheral regions should participate in screening and intervention programs so their chances of presenting a risky development could be minimized.

KEYWORDS: Child development; Evaluation; Primary prevention; Child.

Este artigo é resultado da Dissertação de Mestrado de Andréa Felner Navajas, orientada por Silvana Maria Blascovi-Assis.

1. Mestre em Distúrbios do Desenvolvimento pela Universidade Presbiteriana Mackenzie, São Paulo; Docente da Universidade Paulista, Santos. E-mail: andrea_navajas@hotmail.com

2. Docente do Programa de Pós-Graduação em Distúrbios do Desenvolvimento, Universidade Presbiteriana Mackenzie, São Paulo. E-mail: silvanablascovi@mackenzie.br

Endereço para Correspondência: Silvana Maria Blascovi-Assis. Rua Consolação, 930 - Prédio 28. CEP 01302-907. São Paulo - SP. e-mail: silvanablascovi@mackenzie.br. 


\section{INTRODUÇÃO}

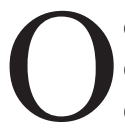
desenvolvimento motor vem sendo estudado por diversos pesquisadores embasados em teorias que norteiam a relação da criança com o seu desenvolvimento neurofisiológico e seu envolvimento com o ambiente. Até meados do século XX, o desenvolvimento motor era considerado sob o prisma da teoria neuromaturacional, para a qual a evolução ocorreria naturalmente a partir da mielinização do sistema nervoso. A teoria dos Sistemas Dinâmicos, bastante aceita hoje em dia, relaciona o desenvolvimento da criança a um conjunto de sistemas que interagem e promovem a aquisição de habilidades específicas, considerando a maturação neurológica como um dos fatores importantes, ressaltando a influência do meio ambiente sobre o mesmo ${ }^{1}$.

Nas últimas décadas, sobretudo nos Estados Unidos e países europeus, os programas de estimulação precoce passaram por transformações. Por este novo enfoque, a estimulação recebeu nova denominação - atenção precoce (do espanhol atención temprana), o que equivale a um conjunto de intervenções dirigidas à população infantil entre zero a seis anos de idade considerando sua família e o contexto em que está inserida, sempre visando à promoção do desenvolvimento infantil ${ }^{2,3}$.

Diversos fatores, porém, podem colocar em risco o curso normal do desenvolvimento de uma criança. Definem-se como fatores de risco uma série de condições biológicas ou ambientais que aumentam a probabilidade de déficits no desenvolvimento neuropsicomotor da criança ${ }^{4}$.

Dentre as principais causas de atraso motor destacam-se o baixo peso ao nascer, a escolaridade materna e as condições socioeconômicas. Quanto maior o número de fatores de risco atuantes, maior será a possibilidade do comprometimento do desenvolvimento ${ }^{5,6}$.

Crianças que se apresentam com risco de atraso, merecem atenção e ações específicas para que problemas motores não se prolonguem na fase adulta. Além disso, atrasos motores frequentemente associam-se a prejuízos secundários de ordem psicológica e social que dificultam a sua socialização e o seu desempenho escolar ${ }^{7}$.

Fatores pré, peri e pós-natais podem influenciar o desenvolvimento e aparecem em grande incidência junto às populações de baixa renda, justificando os procedimentos de acompanhamento do desenvolvimento na primeira infância em crianças de creche ou populações carentes que possam estar expostas a riscos ${ }^{3,8}$.
O desenvolvimento infantil deve ser preocupação de diferentes profissionais da saúde. Compete a esta equipe desempenhar de modo eficiente à intervenção terapêutica. Todas as ações voltadas para a assistência à saúde merecem atenção interdisciplinar ${ }^{9}$. Em especial, fisioterapeutas e terapeutas ocupacionais devem contribuir com as pesquisas sobre o desenvolvimento infantil, especialmente as relacionadas às aquisições motoras, tanto em lactentes saudáveis quanto naqueles expostos a fatores de risco ${ }^{10}$.

Assim, as ações preventivas sobre as alterações do desenvolvimento dependem do conhecimento a respeito da sequência normal e regular das aquisições motoras, que consistirá na base para a elaboração do planejamento do programa de tratamento. O período em que a intervenção é proposta também deve ser considerado. Nos primeiros anos de vida existe uma maior plasticidade cerebral, o que possibilita a otimização de ganhos no desenvolvimento motor. Nesse âmbito, diversas pesquisas demonstraram haver melhora da aquisição de habilidades motoras em crianças que receberam estimulação precoce ${ }^{11}$.

Para o planejamento de uma intervenção eficiente torna-se necessária uma avaliação criteriosa que contemple outros fatores além da impressão clínica. Para a identificação precoce de desvios, tanto do crescimento como do desenvolvimento infantil, diferentes testes e avaliações padronizadas estão descritos na literatura. Estes instrumentos específicos aumentam a taxa de identificação de crianças com suspeitas de atraso e possibilitam o encaminhamento para diagnóstico $\mathrm{e}$ intervenção ${ }^{12}$. A maioria das escalas usadas e reconhecidas por suas propriedades psicométricas foi elaborada em outros países, com características culturais nem sempre semelhantes ao Brasil. Ainda assim, são de grande valia para os estudos em populações brasileiras. Todavia, o uso de escalas nacionais necessita ser mais discutido. A "Escala de desenvolvimento do Comportamento da criança: o primeiro ano de vida" foi desenvolvida pela psicóloga Dra. Elizabeth Batista Pinto, sob orientação dos professores Raymundo Manno Vieira e Luiz Celso Pereira Vilanova ${ }^{13}$. Seu foco é a análise do comportamento da criança brasileira no primeiro ano de vida para ambos os sexos de um a doze meses incompletos. Dotada de fácil aplicação e avaliação, pode ser utilizada por profissionais de diversas especialidades permitindo aos mesmos o planejamento de estratégias de intervenção precoce sempre que o desenvolvimento da motricidade ampla e fina, linguagem e na área pessoal-social mostrar um ritmo lento para a sua idade, além de dar, importantes indícios 
na formulação de hipóteses diagnósticas de crianças com alterações no desenvolvimento do comportamento. Isso pode ser feito através da análise feita por uma ficha de anamnese e a aplicação do teste de sessenta e quatro comportamentos contidos na própria Escala que serão detalhados na metodologia ${ }^{13}$.

Sigolo et al. ${ }^{14}$ dizem ainda que, além de ser padronizada para a população brasileira, esta escala foi desenvolvida exclusivamente para a faixa etária inicial do desenvolvimento; possui material de treino, de capacitação (fita de vídeo denominada Competências do Bebê) e foi aprovada pelo Conselho Federal de Psicologia ${ }^{15}$. Todavia, esse instrumento ainda é pouco utilizado nas pesquisas nacionais.

\section{OBJETIVO}

Este estudo tem como objetivo avaliar o comportamento motor de crianças nascidas a termo na Zona Noroeste de Santos com o uso de um instrumento elaborado e publicado no Brasil, a Escala de Desenvolvimento do Comportamento da criança: o primeiro ano de vida.

\section{MÉTODO}

Participaram desse estudo 50 crianças, sendo 22 meninas e 28 meninos, na faixa etária entre um a doze meses incompletos. Foram critérios de inclusão: residir na Zona Noroeste de Santos; frequentar acompanhamento pediátrico no local onde foi realizada a pesquisa; nascimento a termo, isto é, após trinta e sete semanas completas de gestação. Foram critérios de exclusão: nascimento pré-termo; diagnósticos neurológicos ou presença de síndromes genéticas; malformações congênitas.

$\mathrm{O}$ presente estudo, de caráter descritivo e transversal, foi apresentado ao Comitê de Ética da Universidade Presbiteriana Mackenzie e aprovado sob parecer $n^{\circ} 461 / 12 / 03$. Os participantes receberam a carta de informação e assinaram o termo de Consentimento Livre e Esclarecido.

A unidade de saúde aonde foi feita a pesquisa estava localizada no bairro Jardim Castelo, Zona Noroeste, em Santos. Criada oficialmente em 26 de julho de 1976, a Zona Noroeste completa 40 anos em 2016 e continua se expandindo, tanto no aspecto demográfico, como econômico. A escolha da região se deu em função das características ligadas ao baixo nível socioeconômico.

O projeto foi oferecido pelas secretárias da pediatria no momento em que as mães marcavam as consultas de acompanhamento dos bebês, os quais deveriam ser nascidos a termo entre a faixa etária entre um a doze meses incompletos.

A avaliação foi realizada primeiramente por meio de uma ficha de anamnese que foi respondida pelas mães e/ou responsáveis pelos bebês.

Os participantes foram avaliados pela Escala de Desenvolvimento do Comportamento da Criança no primeiro ano de vida para os sexos feminino e masculino, em uma sala no ambulatório de especialidades da Areia Branca; durante a avaliação as mães foram informadas sobre como se encontrava o desenvolvimento de seus filhos e foram orientadas sobre alguns procedimentos que poderiam facilitar o desenvolvimento dos seus bebês. Os testes foram aplicados em ambiente com temperatura agradável, na presença da mãe ou acompanhante e da pesquisadora, com a criança acordada, alimentada e em boas condições de saúde, durante consultas de rotina para acompanhamento do desenvolvimento no primeiro ano de vida.

Os resultados da padronização da Escala de Desenvolvimento do Comportamento da Criança: o primeiro ano de vida, obtidos na folha de respostas, possibilitam uma avaliação do ritmo de desenvolvimento da criança ${ }^{16}$, considerando-se:

- o comportamento motor em sua classificação quanto ao eixo somático, em comportamento motor axial ou comportamento motor apendicular, e quanto à estimulação, em comportamento motor espontâneo ou comportamento motor estimulado;

- o comportamento atividade, quanto à comunicação, em comportamento atividade não comunicativo ou comportamento atividade comunicativo.

- O ritmo de desenvolvimento do comportamento de criança de um a doze meses pode ser considerado, em cada uma das classificações do comportamento adotadas, com relação à idade:

- Excelente: quando foi observado cada comportamento, obtendo-se "+" em todos aqueles que aparecem (A), normalizam (N) e estabilizam (E);

- Bom: quando foi observado cada comportamento, obtendo-se "+" em todos aqueles que normalizam (N) e estabilizam (E), e "-" apenas em comportamentos que aparecem (A);

- Regular: quando foi observado cada comportamento, obtendo-se nos comportamentos que normalizam (N) tanto "+" como "-", "“+” nos 
que estabilizam (E), e “_" em comportamentos que aparecem (A);

- Derisco: quando foi observado cada comportamento, obtendo-se "+" apenas nos comportamentos que estabilizam (E) e “-” em comportamentos que aparecem (A) e normalizam (N);

- Com atraso: quando foi observado cada comportamento, obtendo-se “-” em comportamentos que aparecem (A), normalizam (N) e estabilizam (E).

\section{RESULTADOS}

As famílias participantes foram compostas em média por dois filhos, sendo que 39 mães eram casadas, 10 eram solteiras e uma era viúva. A escolaridade familiar obtida pela entrevista mostrou que nenhuma das mães era analfabeta, duas tinham nível de primeiro grau incompleto, doze tinham primeiro grau completo, duas não concluíram o segundo grau, 34 tinham o segundo grau completo e nenhuma possuía nível superior completo.

A pesquisa mostrou que das 50 mães, somente 20 trabalhavam fora nas seguintes ocupações: empregada doméstica, auxiliar de escritório, assistente de serviços gerais, manicure, comerciante (lanchonete). Dos 50 pais, 40 trabalhavam fora, os demais eram: um aposentado, um falecido e oito desempregados. As funções que ocupavam: pescador, assistente de serviços gerais, motorista, mecânico, pedreiro, tapeceiro, zelador e ambulante.

A condição de moradia e o tipo de construção foram caracterizadas por 32 famílias que residiam em casas de alvenaria com dois quartos e um banheiro, 12 residiam em edifícios de um ou dois quartos e um banheiro e sete famílias em casas de madeira de um ou dois quartos e um banheiro.

Em relação à gestação, os dados mostraram que as 50 ocorreram a termo (mínimo de trinta e sete semanas e máximo de quarenta e duas semanas). O tipo de parto foi natural para 26 mães, cesariana para 21 e com uso de fórceps para três.

$\mathrm{O}$ peso de nascimento foi entre $2-3 \mathrm{~kg}$ para 18 crianças e acima de $3 \mathrm{~kg}$ para 32. O Apgar não foi informado por 24 mães. Para as demais, o mesmo variou entre sete e dez.

Durante o primeiro ano de vida, seis crianças passaram por períodos de internação por motivos diversos, incluindo icterícia, aspiração do mecônio, imaturidade do sistema respiratório e infecções diversas.
Apenas quatro crianças apresentaram, segundo a mãe, doenças crônicas, entre elas torcicolo congênito, luxação congênita de quadril, pneumonia e anemia.

No grupo estudado 48 crianças foram alimentadas somente com leite materno até os seis meses e duas crianças com leite em pó.

\section{Avaliação a partir da escala de desenvolvimento do comportamento}

As 50 crianças avaliadas foram divididas em três grupos: grupo de desenvolvimento normal (GDN), grupo de desenvolvimento regular (GDR), grupo risco-atraso (GRA). Essa divisão foi proposta para o presente estudo com o objetivo de agrupamento de características comuns entre as crianças com base nos resultados encontrados em cada uma das oito áreas avaliadas, para facilitar a compreensão do ritmo de desenvolvimento dos participantes. Os grupos ficaram, portanto, assim caracterizados:

- Grupo de desenvolvimento normal (GDN): composto por crianças que apresentaram nas avaliações as designações de comportamento excelente e/ou bom para os itens observados na escala adotada;

- Grupo de desenvolvimento regular (GDR): composto por crianças que apresentaram comportamentos classificados em pelo menos um item avaliado como regular, sendo os demais classificados como bom ou excelente;

- Grupo de risco ou atraso (GRA): composto por crianças que obtiveram classificação de risco ou atraso em pelo menos um dos itens avaliados

Os Gráficos de 1 a 5 representam a distribuição das crianças nos respectivos grupos, com identificação de diferenças entre sexos.

Gráfico 1 - Distribuição das crianças nos grupos avaliados

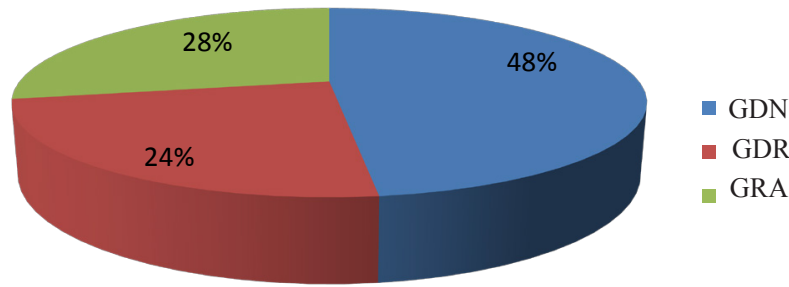


Dentro do grupo de desenvolvimento normal (GDN), equivalente a doze crianças no total, $50 \%$ eram do sexo masculino, e $50 \%$ do sexo feminino. O Gráfico 2 representa a essa distribuição.

Gráfico 2 - Valores de incidência quanto ao sexo em GDN

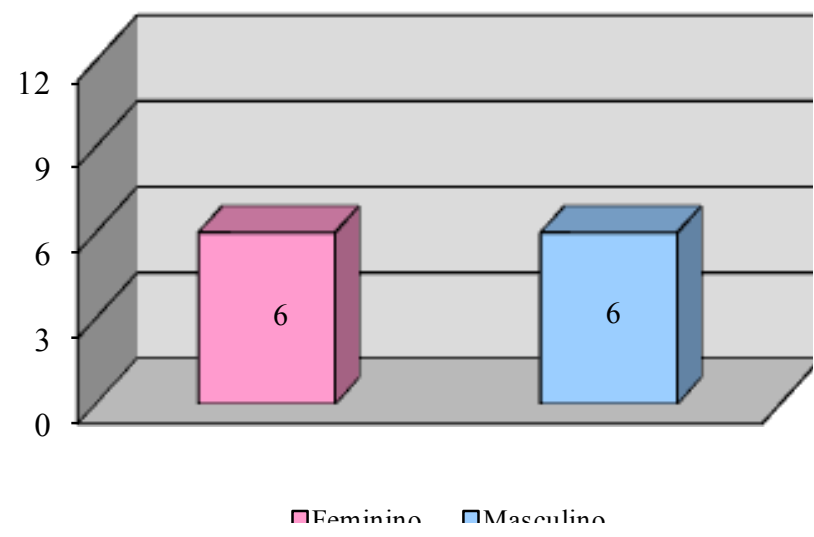

O grupo de desenvolvimento regular (GDR), representado no Gráfico 3 é composto por quatorze crianças no total, sendo $42,8 \%$ do sexo feminino e $57,1 \%$ do sexo masculino.

Gráfico 3 - Valores de incidência quanto ao sexo em GDR

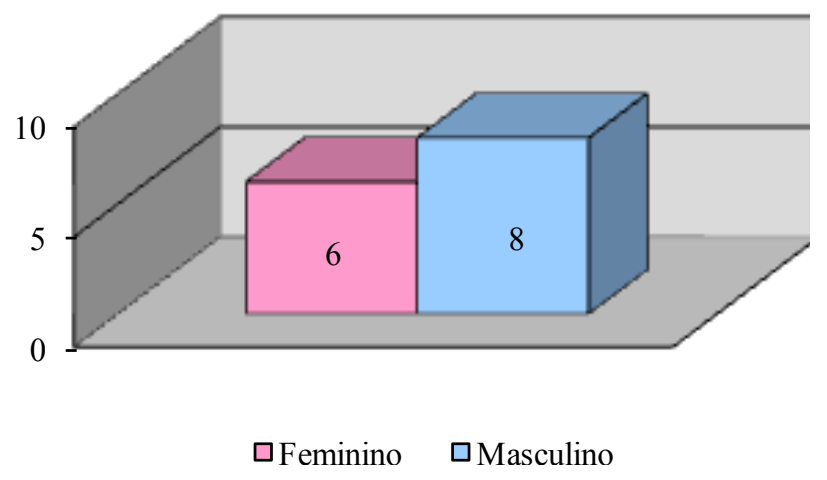

Já no grupo de desenvolvimento risco-atraso (GRA), estão contidas vinte e quatro crianças dentre as avaliadas no total, sendo que seis fazem parte do subgrupo risco e dezoito estão no subgrupo atraso, demonstrados no Gráfico 4.

Gráfico 4 - Representação dos subgrupos dentro do GRA

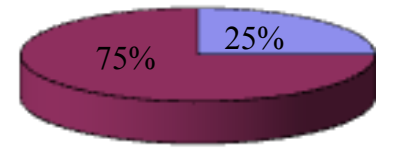

\section{$\square$ Risco $\square$ Atraso}

O subgrupo risco foi representado por três meninas $(50 \%)$ e três meninos $(50 \%)$, enquanto que o subgrupo atraso foi composto por sete meninas $(38,8 \%)$ e onze meninos $(61,2 \%)$, representados no Gráfico 5.

Gráfico 5 - Incidência quanto ao sexo no subgrupo risco e atraso

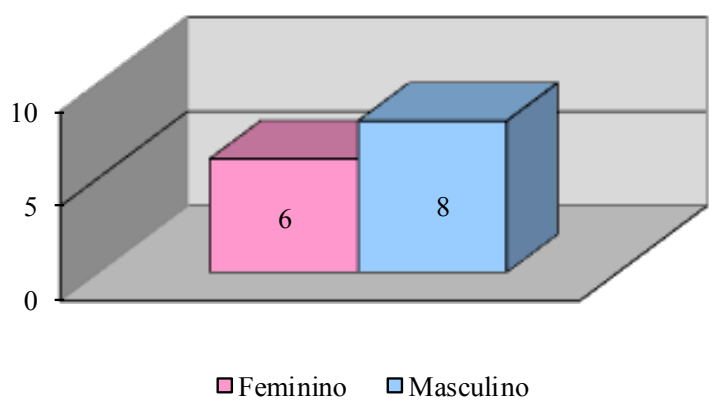

\section{DISCUSSÃO}

A atenção integral à saúde da criança deve ser foco primordial nos programas comunitários, considerando que a prevenção primária deve garantir o acompanhamento regular e o oferecimento de condições e orientações para que a mesma se desenvolva e possa otimizar seus potenciais. Este seguimento deve contar com o apoio da família, da comunidade e dos profissionais envolvidos com a saúde e a educação, favorecendo o bem-estar familiar ${ }^{17}$. 
A região onde foi realizado este estudo caracteriza-se pelo grande número de habitantes residentes em favelas, que estão, por consequência, mais sujeitos às doenças e à violência. $\mathrm{O}$ índice de mortalidade infantil é bastante alto, revelando a necessidade da prevenção em diferentes esferas dos cuidados à saúde desta população. Esses dados estão de acordo com a literatura que refere o nível socioeconômico como fator de risco ao desenvolvimento ${ }^{4,5,6}$

$\mathrm{O}$ grupo aqui avaliado apresentou algumas características que confirmam a relevância da escolha da região estudada. A maioria residia em casas de alvenaria (32), edifícios (11) e casas de madeira (7), porém uma das limitações do questionário aplicado refere-se à falta de informação sobre o espaço domiciliar, ou seja, se o mesmo era favorável às condições para exploração do ambiente e realização de brincadeiras infantis. Muitas dessas moradias eram provenientes de projetos feitos pela Companhia de Habitação da Baixada Santista (COHAB) e Companhia de Desenvolvimento Habitacional Urbano (CDHU) em parceria com a prefeitura. Outras lacunas foram observadas durante a aplicação da ficha de anamnese. Outras informações relevantes também não foram disponibilizadas, representando uma limitação do estudo, tais como a estrutura familiar (renda total, administração da renda, mudanças de residência); características da dinâmica familiar (relação família/ sociedade, família/ parentes, família/ vizinhos, lazer/ comunidade); relação do casal (harmonia, problemas quanto aos filhos, ordem econômica, sexo, objetivos de vida, temperamento, educação dos filhos), entre outros aspectos.

Apesar da maioria dos pais ter o segundo grau completo (34) mães e (30) pais, as atividades profissionais eram exercidas em pontos de comércio e serviços de pequeno porte, tais como; bazares, supermercados, padarias, oficinas mecânicas, lojas de materiais de construção, barcos de pescaria, auxiliar de serviços gerais, serviços que remetem à baixa renda. Observa-se que a escolaridade tem sido apontada como fator de risco ${ }^{18,19}$, porém o grupo avaliado, apesar de não ser caracterizado por pais com baixo nível de escolaridade apresentou grande percentual de risco, talvez mais associado às

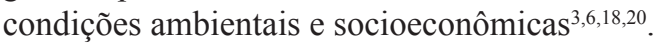

Os resultados encontrados mostram que cerca de $48 \%$ das crianças avaliadas (24 apresentavam atraso em alguma das áreas do desenvolvimento, ou em zona de risco para o desenvolvimento. Pode-se considerar este índice bastante alto, já que os critérios de exclusão previam a não participação de crianças que apresentassem a priori, características que pudessem levar ao atraso em qualquer área. O elemento comum no grupo estudado era a residência na Zona Noroeste da cidade de Santos.

A influência do ambiente no desenvolvimento da criança embasada nas teorias de desenvolvimento, como por exemplo, a Teoria dos Sistemas Dinâmicos onde o desenvolvimento é considerado dependente de atividades e exigências do meio ambiente, realçando as propriedades auto-organizadoras do sistema sensório-motor ${ }^{1}$.

Saccani et al. ${ }^{18}$ relacionam o desenvolvimento humano com efeitos advindos da família, do meio ambiente e da sociedade. Esses autores afirmam que problemas biológicos podem ser modificados por fatores ambientais e que determinadas situações de vulnerabilidade podem ter etiologia relacionada a fatores sociais e do meio ambiente. A associação de baixa renda familiar, baixo nível de escolaridade e de longo período de privações exerce efeito negativo sobre o desenvolvimento infantil.

Outros autores examinaram a influência do ambiente domiciliar no desenvolvimento de habilidades motoras, e os resultados mostraram que crianças aos 18 meses e aos 5 anos, consistentemente apresentaram piores desempenhos nas habilidades motoras grossas ${ }^{20}$. Por outro lado, estudos consideraram que fatores sociodemográficos, portanto ambientais, estão mais associados ao desempenho motor fino ${ }^{21}$.

A importância das condições socioeconômicas familiares no desenvolvimento cognitivo das crianças foi confirmada em outros estudos. Em um deles, pesquisadores avaliaram crianças aos 5 anos de idade, considerando a baixa renda familiar, a duração das privações e a escolaridade materna. A associação de baixa renda familiar, baixo nível de escolaridade e de longo período de privações exerce efeito negativo sobre o desenvolvimento infanti1 ${ }^{18,19}$. Estudos realizados com crianças coreanas desnutridas, adotadas em lares americanos, demonstraram que elas se desenvolveram satisfatoriamente com o enriquecimento ambiental, desde que o estímulo fosse anterior aos 2 anos de idade e oferecido por um período suficientemente longo ${ }^{22}$.

A atuação familiar vai além dos pais, pode incluir outros parentes, cuidadores e até mesmo a comunidade, possibilitando o desenvolvimento de estratégias de estimulação a partir do espaço vivido da criança. Sem o envolvimento familiar, sugerem os autores, a intervenção tende a não obter sucesso, e os poucos efeitos atingidos ficam propensos a desaparecer quando a intervenção é descontinuada. Destaca-se, assim, o processo de interação da criança em seu ambiente (o organismo, o lar, a creche, a escola, a vizinhança) como mecanismo responsável pelo seu desenvolvimento ${ }^{23}$.

A avaliação do desenvolvimento do comportamento através da Escala de Desenvolvimento do Comportamento 
da Criança - EDCC pode favorecer a deteç̧ão precoce de atrasos e dificuldades no desenvolvimento do comportamento e contribuir para prevenir os efeitos prejudiciais na criança, seja no planejamento detalhado de estratégias de estimulação, no encaminhamento para especialistas ou no aprimoramento do conhecimento e da sensibilidade parental sobre o desenvolvimento da criança com uma possível redução dos distúrbios na interação pais/ criança $^{13,14}$. Além disso, como um benefício adicional, a avaliação do bebê utilizando a Escala de Desenvolvimento do Comportamento da Criança - EDCC na presença dos pais, pode ser uma forma preventiva de intervenção, tendo em vista que possibilita aos pais conhecer melhor as competências e características do comportamento do seu bebê, habilitando-os possivelmente a responder melhor às necessidades adaptativas da criança e a estabelecer com a ela uma interação mais satisfatória ${ }^{13}$.

Almeida et al. ${ }^{24}$ realizaram um estudo com o objetivo de investigar a influência de um programa de intervenção motora no comportamento do bebê no terceiro trimestre de vida em creches de baixa renda. Tarefas de perseguição visual, manipulação do brinquedo e do controle postural do bebê foram implementadas no programa interventivo. As avaliações dos bebês foram realizadas com a aplicação da Escala do Desenvolvimento do Comportamento da Criança no Primeiro Ano de Vida ${ }^{16}$, no início e ao final do período interventivo. Os resultados sugeriram que os bebês do Grupo Interventivo $(n=20)$ demonstraram desempenho significativamente superior no pós-intervenção em relação ao Grupo Controle ( $\mathrm{n}=20)$ e, também, ganhos positivos do pré para o pós-intervenção. No entanto, mudanças positivas e significativas não foram observadas no Grupo Controle. Concluiram que, programas de avaliações e intervenções motoras propiciam mudanças positivas no comportamento de bebês no terceiro trimestre em creches de baixa renda.

\section{REFERÊNCIAS}

1. Shumway-Cook A, Woollacott MH. Controle postural. In: Shumway-Cook A, Woollacott MH, editores. Controle motor: teoria e aplicações práticas. São Paulo: Manole; 2003 p.2-78.

2. Dunst C, Bruder MB. Valued outcomes of service coordination, early intervention and natural environments. Exceptional Children. 2002;68(3):361-75. doi: 10.1177/001440290206800305
Estudos que indiquem riscos ao desenvolvimento infantil devem ser considerados na elaboração de Políticas Públicas para que qualquer fator que possa comprometer as condições de crescimento e desenvolvimento na infância seja minimizado. Brito et al. ${ }^{25}$ ressaltam a necessidade de cuidados com a observação e acompanhamento do desenvolvimento nos primeiros cinco anos de vida, para que possíveis alterações não sejam percebidas tardiamente. Para os autores, a intervenção precoce deve pensada para atenuar lacunas do desenvolvimento para as crianças nesta faixa etária.

Foi possível concluir que as crianças avaliadas neste estudo apresentaram significativo atraso no seu desenvolvimento, apesar terem nascido a termo, com bom peso ao nascimento, Apgar satisfatório, terem sido amamentadas pela mãe até os seis meses e serem filhos de mães de nível cultural médio. Acredita-se que os fatores contribuintes para os resultados encontrados estejam fundamentados no contexto socioambiental dessas crianças. $\mathrm{O}$ ambiente físico, o contexto relacional famíliamãe-criança, contexto social (comunidade com quem se relaciona).

$\mathrm{O}$ instrumento utilizado mostrou-se sensível para detectar alterações no comportamento motor do grupo avaliado. Todavia, torna-se cada vez mais relevante ampliar a concentração de esforços para a tríade composta por criança-família-sociedade e não apenas na atenção à criança em desenvolvimento, a fim de privilegiar a eficácia nas ações preventivas para a implementação de projetos de esclarecimento e orientação à comunidade, às famílias das gestantes, à conscientização das gestantes sobre o desenvolvimento motor normal da criança e a estimulação adequada as suas etapas. Para tanto, avaliações periódicas dos bebês junto às mães, orientações quanto ao manuseio e mobilização da criança desde o nascimento e palestras sobre o brincar e a influência do ambiente no desenvolvimento biopsicossocial seriam fundamentais para a qualidade do desenvolvimento de populações carentes.

3. Soejima CS, Bolsanello MA. Programa de intervenção e atenção precoce com bebês na educação infantil. Educ Rev. 2012;(43):65-79. doi: 10.1590/S0104-40602012000100006.

4. Miranda MC, Pompéia S, Bueno OFA. Um estudo comparativo das normas de um conjunto de 400 figuras entre crianças brasileiras e americanas. Rev Bras Psiquiatr. 2004;26(4):226-33. doi: 10.1590/S151644462004000400005 . 
5. Halpern R, Giugliani ERJ, Victoria CG, Barros FC, Horta BL. Fatores de risco para a suspeita de atraso no desenvolvimento neuropsicomotor aos 12 meses de vida. Rev Chil Pediatr. 2000;7(6)421-8. doi: 10.4067/S037041062002000500016.

6. Assis-Madeira EA, Carvalho SG, Blascovi-Assis SM. Desempenho funcional de crianças com paralisia cerebral de níveis socioeconômicos alto e baixo. Rev Paul Pediatr (São Paulo). 2013;31(1)51-7. doi: 10.1590/S010305822013000100009.

7. Willrich A, Azevedo CCF, Fernandes JO. Desenvolvimento motor na infância: influência dos fatores de risco e programas de intervenção. Rev Neurocienc. 2009;17(1):51-6.

8. Brito AG. Prevención en el âmbito de las poblaciones de risgo biológico. In: Perez-López J, Brito AG, editores. Manual de atención temprana. Madrid: Pirámide; 2004

9. Alvim CG, Guimarães FG, Meinberg NLS, Aguiar LT, Caetano LCG, Carrusca LC, Caetano LM, Labanca L, Fonseca NM, Paulo RAM, Tagliaferri TL, Oliveira Junior HA, Alves ACS, Sousa AZA. A avaliação do desenvolvimento infantil: um desafio interdisciplinar. Rev Bras Educ Med. 2012;36(1 supl.1):51-6. doi: 10.1590/ s0100-55022012000200007.

10. Santos DCC, Campos D, Gonçalves VMG, Mello BBA, Campos TM, Gagliardo HGRG. Influência do baixo peso ao nascer sobre o desempenho motor de lactentes a termo no $1^{\circ}$ semestre de vida. Rev Bras Fisioter. 2004;8(3):2616. Disponível em: http://rbf-bjpt.org.br/files/v8n3/v8n3a12. pdf.

11. Blauw CH, Hadders MA. Systematic review of the effects of early intervention on motor development. Dev Med Child Neurol. 2005;47(6):421-32. Disponível em: https://www. ncbi.nlm.nih.gov/pubmedhealth/PMH0022089/.

12. Campos D, Santos DCC, Gonçalves VMG, Goto MMF, Arias AV, Brianeze ACGS. Agreement between scales for screening and diagnosis of motor development at 6 months. J Pediatr (Rio J) 2006:82(6):470-4. doi: 10.2223/ JPED.1567.

13. Batista Pinto E. O desenvolvimento do comportamento do bebê prematuro no primeiro ano de vida. Behavior development of preterm infants in the first year of life. Psicol Reflex Crit (Porto Alegre). 2009;22(1)76-85. doi: 10.1590/S0102-79722009000100011.

14. Sigolo AR, Aiello ALR. Análise de instrumentos para triagem do desenvolvimento infantil. Paidéia (Ribeirão Preto). 2011;21(48)51-60. doi: 10.1590/S0103$863 X 2011000100007$.

Recebido em: 01.02.15

Aceito em: 12.09 .16
15. Batista Pinto E. La psychothérapie mère/bébé: un abordage de l'enfant prématuré. Symposium conducted at the meeting of the World Association of Infant Mental Health Congress - WAIMH. Montréal, Canada; 2000.

16. Batista Pinto E, Vilanova LCP, Vieira RM. O desenvolvimento do comportamento da criança no primeiro ano de vida: padronização de uma escala para a avaliação e o acompanhamento. São Paulo, SP: Fundação de Amparo à Pesquisa do Estado de São Paulo; 1997.

17. Falbo ECP, Andrade RD, Furtado MCC, Mello DF. Estímulo ao desenvolvimento infantil: produção do conhecimento em enfermagem. Rev Bras Enferm. 2012;65(1):148-54. doi: 10.1590/S0034-71672012000100022.

18. Saccani R. Brizola E. Giordani AP. Bach S, Resende TL, Almeida CS. Avaliação do desenvolvimento neuropsicomotor em crianças de um bairro da periferia de Porto Alegre. Scientia Med. 2007;17(3):130-7.

19. Duncan JD, Brooks-Gunn, J, Klebanov PK. Economic deprivation and early childhood development. Child Dev. 1994;65(2):296-318.

20. Oliveira GE, Magalhães LM, Salmela LFT. Relação entre muito baixo peso ao nascimento, fatores ambientais e o desenvolvimento motor e o cognitivo de crianças aos 5 e 6 anos. Rev Bras Fisioter. 2011;15(2):138-45. doi: 10.1590/ S1413-35552011000200009.

21. Chen PS, Jeng SF, Tsou KI. Developmental function of very-low-birth weight infants and full-term infants in early childhood. J Formos Med Assoc. 2004;103(1):23-31.

22. Lien NM, Meyer KK, Winick M. Early malnutrition and "late" adoption: a study of their effects on the development of Korean orphans adopted into american families. Am J Clin Nutr. 1977;30(10):1734-9.

23. Fadden G. Training and disseminating family interventions for schizophrenia: developing family intervention skill with multi-disciplinary groups. J Fam Ther. 2006;28(1):23-38. doi: 10.1111/j.1467-6427.2006.00335.x.

24. Almeida CS, Valentini NC, Lemos CXG. A influência de um programa de intervenção motora no desenvolvimento de bebês em creches de baixa renda. Temas Desenvolv. 2006;14(83/84):40-8.

25. Brito CML, Vieira GO, Costa MC, Oliveira NF. Desenvolvimento neuropsicomotor: o teste de Denver na triagem dos atrasos cognitivos e neuromotores de pré-escolares. Cad Saúde Pública (Rio de Janeiro). 2011;27(7):1403-14. doi: 10.1590/S0102$311 \times 2011000700015$. 